\title{
PHYSICOCHEMICAL AND BACTERIOLOGICAL PROPERTIES OF SURFACE WATERS FROM TWO LOCALITIES IN RIVERS STATE, NIGERIA
}

\section{Michael Adetope Aladese* and Juliana Okwena Pondei}

\author{
Department of Microbiology Federal University Otuoke, Nigeria
}

*Corresponding author E-mail: aladesema@gmail.com; Tel: +2348066417988

Cite this article:

Aladese, M.A. and Pondei, J.O. (2021), Physicochemical and Bacteriological Properties of Surface Waters from two Localities in Rivers State, Nigeria. African Journal of Environment and Natural Science Research 4(2), 39-58. DOI: 10.52589/AJENSR9Y0AMSK8.

\section{Manuscript History}

Received: 30 March 2021

Accepted: 21 April 2021

Published: 27 April 2021

Copyright $\odot 2020$ The Author(s). This is an Open Access article distributed under the terms of Creative Commons AttributionNonCommercial-NoDerivatives 4.0 International (CC BY-NC-ND 4.0 ), which permits anyone to share, use, reproduce and redistribute in any medium, provided the original author and source are credited.

\begin{abstract}
This study investigated the physicochemical and bacteriological properties of surface waters from Bori and Okoli'ile towns in Rivers State, Nigeria. A total of 360 brackish water samples were collected from January to December, 2019 from the two localities. Samples were subjected to physicochemical, trace elements and bacteriological examinations. Isolated colonies from microbiological examinations were subjected to further biochemical and physiological characterization. The result showed $\mathrm{pH}$ mean values were higher in the month of January to March, a period where rainfall is less frequent (dry months). Similar trend of occurrence was observed in the monthly mean values of temperature $\left({ }^{0} \mathrm{C}\right)$ and salinity $(\mathrm{mg} / \mathrm{L})$. Most of the physicochemical indices of water quality measurement recorded average values exceeding the stipulated acceptable environmental tolerance limits by the World Health Organisation (WHO). The microbiological examinations showed lower monthly mean values of total heterotrophic count, total coliforms and faecal coliforms counts during the wet months with statistically significant difference $(P<0.05)$ when compared to the average values obtained in the dry months. The Pearson's correlation coefficient showed high relationship between total heterotrophic count and faecal coliforms, an indication of increase in human wastes into aquatic environments leading to higher bacterial population within the waterbody, which could be a predisposing factor for epidemic. It is recommended that local and regional governments should enforce appropriate measures to encourage personal and community practice of hygiene.
\end{abstract}

KEYWORDS: Physicochemical Analysis, Bacteriological Properties, Surface Waters. 


\section{INTRODUCTION}

Water undoubtedly is the most essential substance for the maintenance of life (Oyinloye \& Jegede, 2004). The component of a living cell is largely made up and maintained by water. About $71 \%$ of earth's surface area is made up of water (Okeola et al., 2010). Water exists in different forms which could be classified as groundwater and surface water. Groundwater includes hand-dug wells, boreholes and springs (NBS, 2012). Examples of surface water are streams, rivers, lakes, ponds, creeks, seas and oceans (Higler, 2012). Furthermore, water could be classified based on the salinity; these are fresh water (absence or minimal saline content), brackish water (moderate salinity) which are creeks, estuaries and lagoons, and marine water (considerably high amount of salinity content) with examples being seas and oceans.

Water quality is simply the assessment of the physical and chemical properties of a water body, both of which are collectively referred to as physicochemical indices of water quality assessment. Physicochemical properties of water entail physical examination, presence of ions, trace elements and heavy metals. Typical examples of physicochemical parameters are $\mathrm{pH}$, salinity, electrical conductivity, dissolved solids, turbidity, total alkalinity and dissolved oxygen. Investigation of ions (nitrates, sulphates and phosphates) and trace elements like iron, zinc, manganese, cadmium, calcium, magnesium, nickel, copper, vanadium and chromium make up the complete physicochemical investigation of water quality (Diersong et al., 2009).

Additionally, water quality assessment involves microbiological examinations. This includes the investigation for total heterotrophic count, total coliform, faecal coliform, Escherichia coli, Enterococcus faecalis and fungi (Cheesbrough, 2006). The bacteria used in this water quality assessment are collectively referred to as indicator microorganisms (Jay et al., 2007).

Surface water is an integral part of life to the people of the Niger-Delta region. It serves as a source of drinking water, fishing activities, agricultural activities, manufacturing of local products, recreational activities and a means of transportation within the creek communities. The quality of these surface waters has a direct positive and detrimental effect on the aquatic ecosystem and human population residing in the vicinity.

This study was conducted in two settlements in Rivers State, South-South Nigeria with the aim to determine the physicochemical and bacteriological qualities of surface waters within these selected localities. The objective of this study is to ascertain the level of chemical and biological contamination of these water bodies, and possibly recommend ways to avert possible outbreaks of epidemics through changes in unhealthy practices.

\section{MATERIALS AND METHODS}

\section{Study areas}

Bori $\left(4^{0} 40^{\prime} \mathrm{N} ; 7^{0} 22^{\prime} \mathrm{E}\right)$ is the second largest town in Rivers State after Port Harcourt, SouthSouth of Nigeria. Bori is the commercial centre of the Ogoni-land. The town is bordered by several creek communities like Gokana, Khana, Yeghe, Kor and Andoni communities. The people of Bori engage in farming and fishing as their primary sources of occupation.

Okoli'ile ( $\left.4^{0} 31^{\prime} \mathrm{N} ; 7^{0} 27^{\prime} \mathrm{E}\right)$ which could also be called Okolo'ile is one of the settlements in the heart of Andoni-land of Rivers State. Okoli'ile is a creek community with its inhabitants 
predominantly speaking the obolo dialect. Okoli'ile is bordered by numerous riverine communities like Ebukuma, Ngo, Uyeada and Ikuru town. The indigenous population are primarily involved in fishing and farming practices.

\section{Sampling of water}

A total of 360 brackish water samples were collected from the two study settlements. Fifteen (15) samples were collected from five (5) locations each in the two areas. Samples were collected for the duration of 12 months spanning from January to December 2019. Water samples were collected into sterile 5L plastic containers, placed on ice and transferred to the laboratory for analysis within $24 \mathrm{hrs}$. Manganous sulphate solution $(1 \mathrm{ml})$ and sodium iodideazide were added on $300 \mathrm{ml}$ opaque specifically designed to collect water samples for dissolved oxygen. These chemicals were added to fix oxygen when collected on site.

\section{Analysis of physicochemical parameters}

All physicochemical properties of collected samples from Bori and Okoli'ile were determined based on the procedure elucidated by American Public Health Association (APHA, 1992). All parameters, except otherwise stated, were measured in $\mathrm{mg} / \mathrm{L}$ and data obtained were compared with World Health Organisation (WHO) standards for environmental acceptability.

\section{Analysis of heavy metals}

All heavy metals and trace elements were measured in $\mathrm{mg} / \mathrm{L}$. Heavy metals analyses were carried out using atomic absorption spectrophotometer (thermal elemental 969 series). The procedures for the analysis of heavy metals and trace elements used in this study were based on the methods described by Ademoroti, 1997. Data obtained were compared with WHO standards for environmentally acceptable limits of tolerance.

\section{Bacteriological examination of water samples}

Determination of bacterial profiling was performed based on the described methods of APHA, 2005. The total bacterial counts were determined with serial dilution up to $10^{-6}$. The most probable number (MPN) in the procedure for sanitary analysis of water was employed to determine the total and faecal coliform enumeration. Further identification for specific species was done using IMVIC test as described by APHA (2005). Isolated colonies were subjected to various selective media and biochemical tests which are Gram's reactions, oxidase, triple sugar iron fermentation, sucrose utilization and motility test.

\section{Statistical analysis}

All data obtained were represented in the forms of means with their standard deviations and pictorial representation using Microsoft excel version 2016. The statistical comparisons of means were also determined using Microsoft excel version 2016. Pearson's correlation coefficients were calculated using IBM SPSS (version 23.0). 


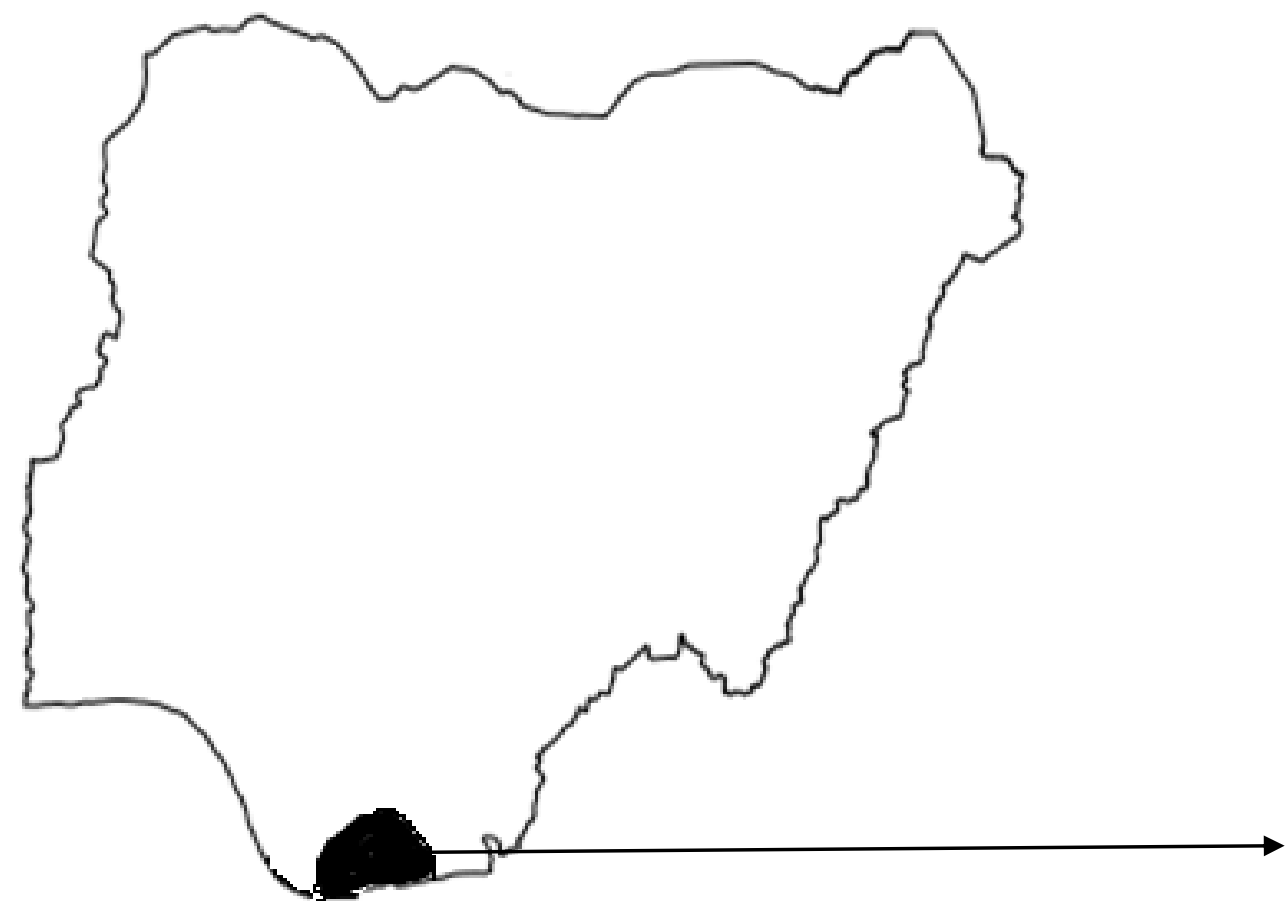

Figure 1a. Map of Nigeria showing the region where localities were situated.

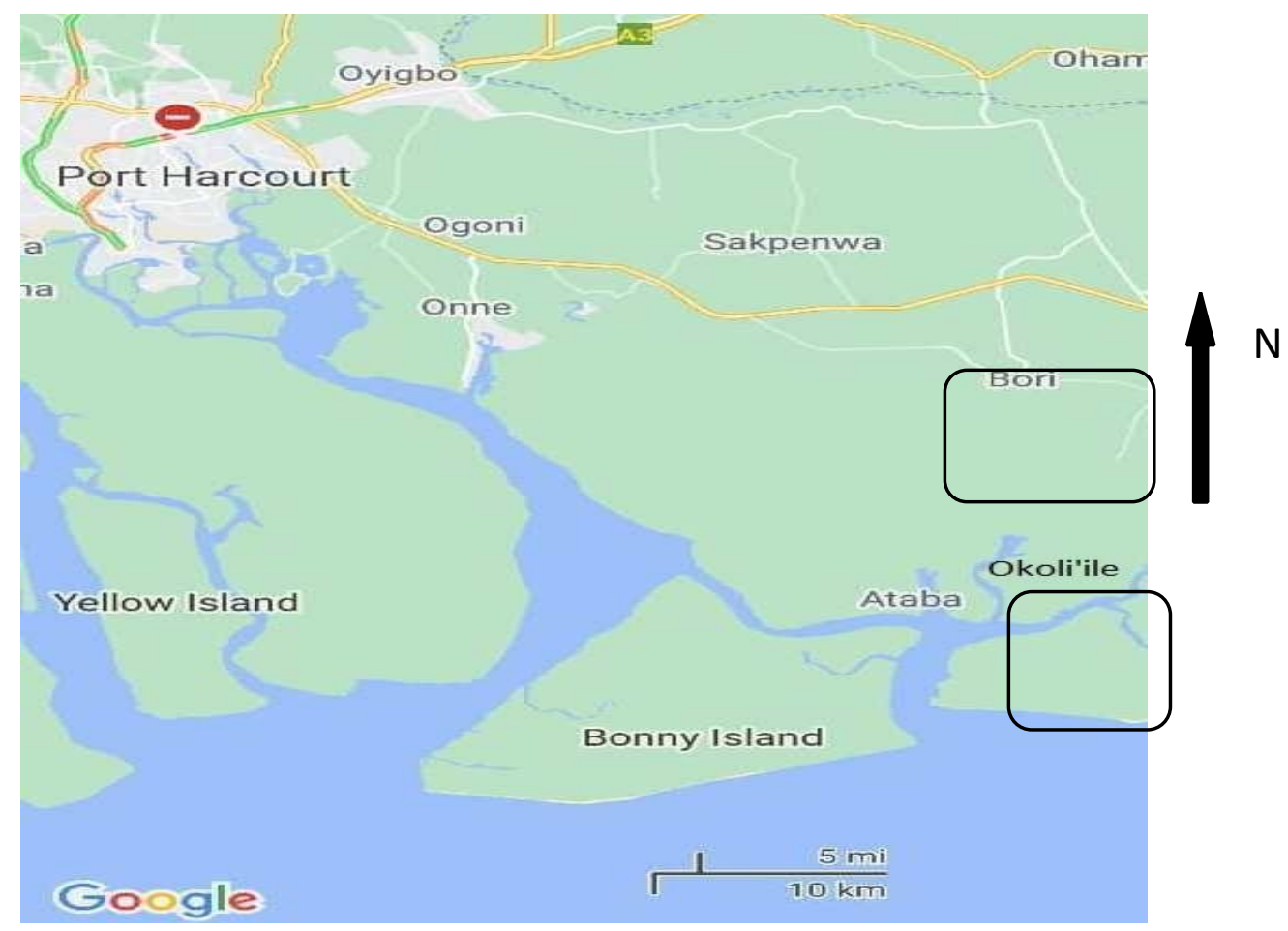

Figure 1b. Map of sections of Rivers State showing the two settlements of study. Source: Google maps inc. 


\section{RESULT}

The graphical representation of the obtained mean values of $\mathrm{pH}$ of brackish water samples in Bori and Okoli'ile showed higher average figures in the month of January to March while lower values were observed from May to October (figure 2). The statistical students' t-test comparison of mean values in the two localities show no significant difference $(\mathrm{P}>0.05)$. The pictorial representation of monthly mean values of temperature $\left({ }^{0} \mathrm{C}\right)$ showed lower temperature values in the month of May to October (figure 3). The statistical comparison of the mean values using statistical students' t-test revealed no statistically significant difference $(\mathrm{P}>0.05)$ in the values from the two localities. The evaluation of salinity content of the brackish water samples from the two areas showed reduced concentration of salinity $(\mathrm{mg} / \mathrm{L})$ from April to October in Okoli'ile, while there was slight reduction in the concentration from June to December in Bori (figure 4). The statistical comparison of means of salinity concentrations showed statistically significant differences $(\mathrm{P}<0.05)$.

The monthly mean values of the physicochemical parameters of brackish water revealed most of the environmental indices of quality assessment of water in Bori exceeded the WHO acceptable limits (table 1). Prominent are electrical conductivity $(\mu \mathrm{S} / \mathrm{cm})$, dissolved solids, turbidity and hardness of water which recorded values much higher than the acceptable limits of tolerance. The monthly average values of trace elements and heavy metals from Bori (table 2) showed elements like $\mathrm{Ca}^{2+}, \mathrm{Mg}^{2+}$ and $\mathrm{Fe}^{2+}$ exceeded the limits. The similar trends of occurrence were noticed in the values of physicochemical parameters (table 3) and trace elements (table 4) in Okoli'ile.

The microbiological examination of brackish water samples from Bori and Okoli'ile entailed the investigation of the total heterotrophic count (figure 5). It was observed that in the month of April to September, there were lowered recorded mean values. Statistical comparisons of means showed Okoli'ile to be significantly higher at $\mathrm{P}<0.05$ using the students' t-test. Similarly, lower monthly average values were observed in the month of April to September (figure 6). There is statistical significance in the distribution of the monthly mean values $(\mathrm{P}<0.05)$ with values from Okoli'ile having statistically higher values. In the same vein, the monthly mean values of faecal coliform counts from Bori and Okoli'ile (figure 7) showed lowered values from April to October. The monthly mean values obtained from Okoli'ile was statistically higher $(\mathrm{P}<0.05)$ when compared to the values of faecal coliform counts from Bori. Table 5 shows the monthly distribution of values of Enterococcus faecalis from the two settlements with statistical significance in the mean from Okoli'ile.

The result of the Pearson's correlations of indicator microorganisms and three (3) physicochemical parameters in Bori (table 3 ) was investigated. There was a high correlation in the distribution of total heterotrophic count with total and faecal coliforms. Similar trend was observed in table 7 which showed Pearson's correlation coefficient of indicator microorganisms with physicochemical parameters from Okoli'ile's brackish water. 
African Journal of Environment and Natural Science Research

ISSN: 2689-9434

Volume 4, Issue 2, 2021 (pp. 39-58)

www.abjournals.org

7.45

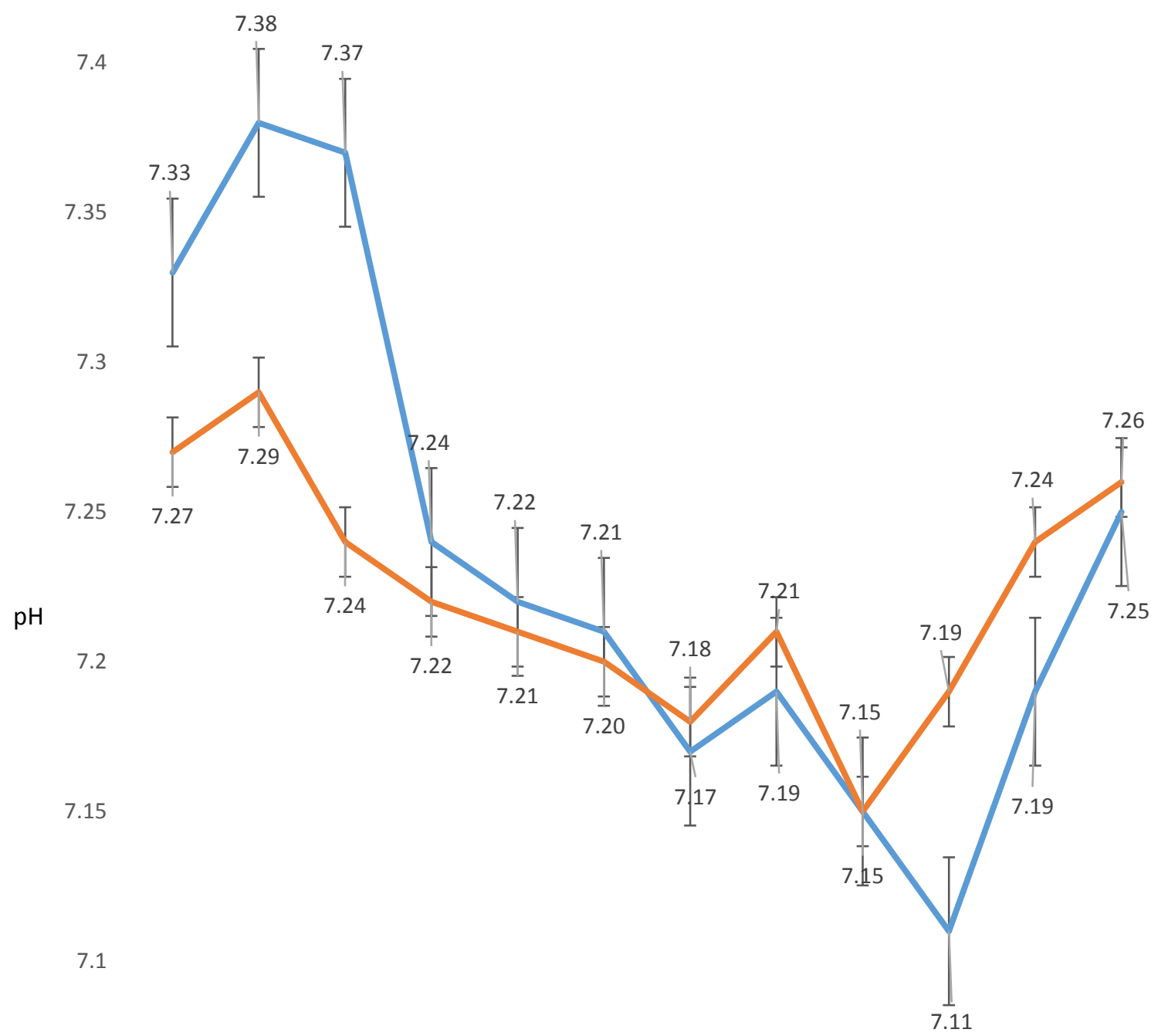

7.05

Jan Feb Mar Apr May Jun Jul Aug Sep Oct Nov Dec

Months

$\longrightarrow \mathrm{pH}$ (Bori) $\longrightarrow \mathrm{pH}$ (Okoli'ile)

Figure 2. Monthly mean values of pH of Brackish water samples in Bori and Okoli'ile, Rivers State 
African Journal of Environment and Natural Science Research

ISSN: 2689-9434

Volume 4, Issue 2, 2021 (pp. 39-58)

www.abjournals.org

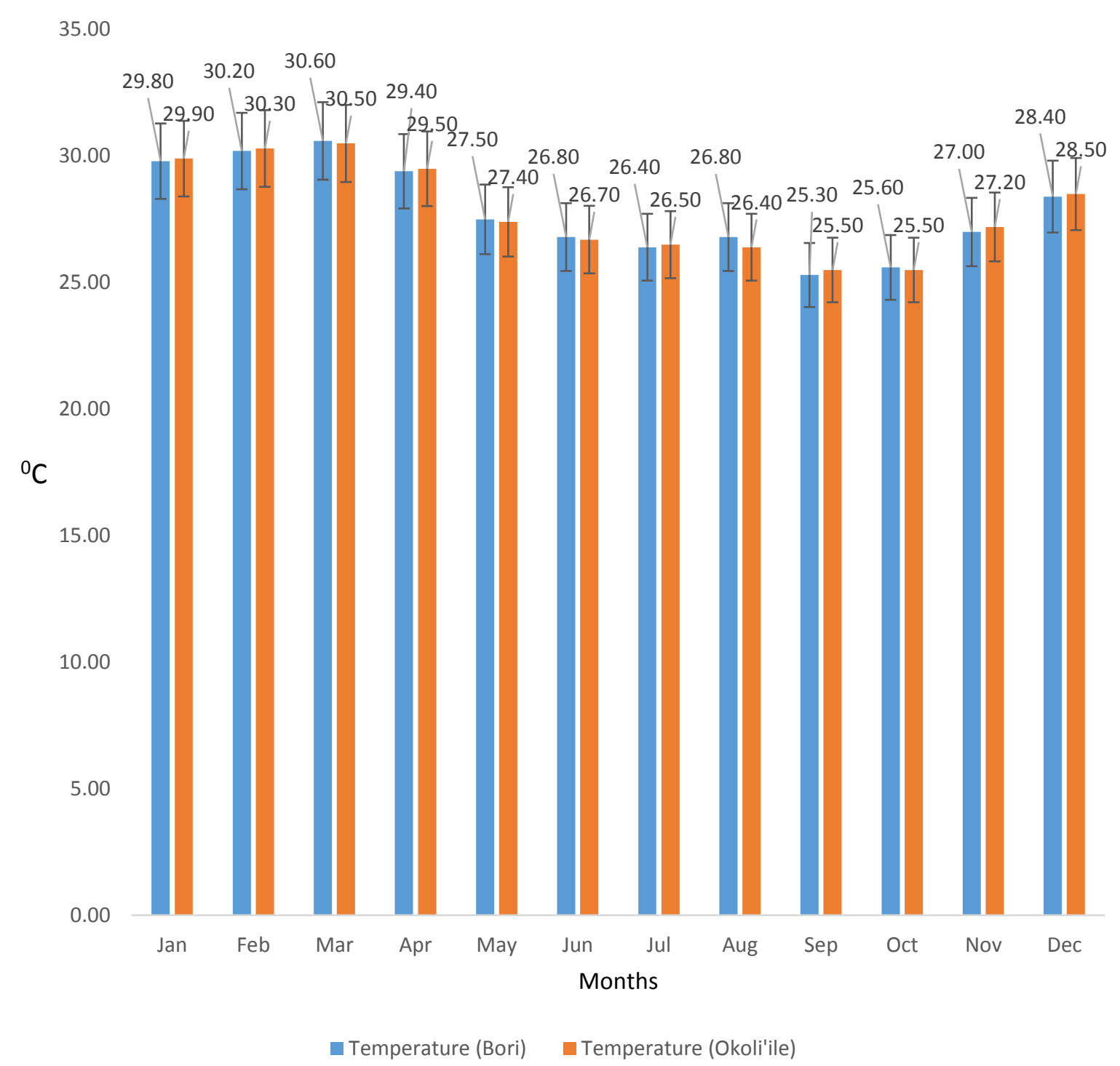

Figure 3. Monthly mean values of Temperature $\left({ }^{\circ} \mathrm{C}\right)$ of Brackish water samples in Bori and Okoli'ile, Rivers State 


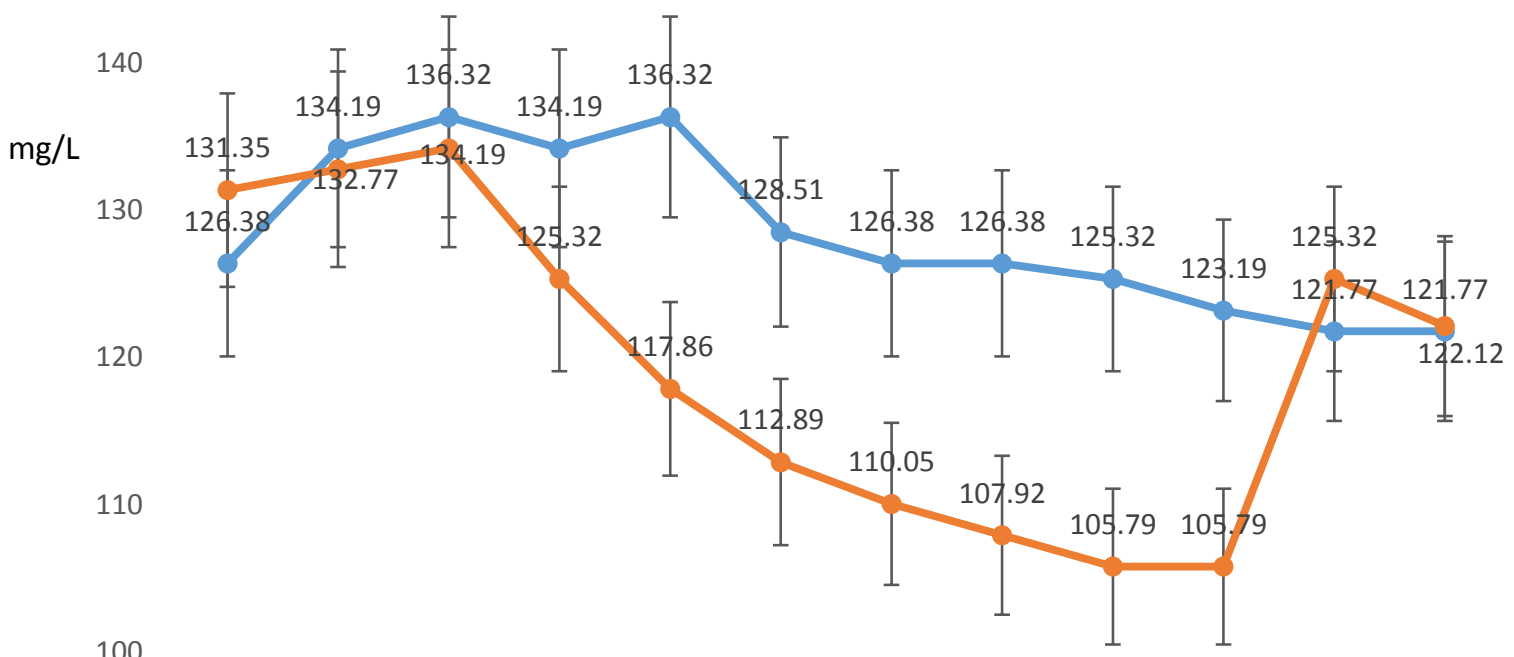

100

90

80

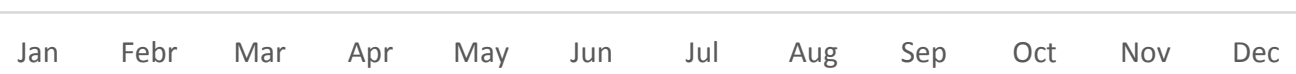

Months

$\longrightarrow$ Salinity (Bori) $\quad-$ Salinity (Okoli'ile)

Figure 4. Monthly mean values of salinity (mg/l) of Brackish water samples in Bori and Okoli'ile, Rivers State 


\section{Table 1: Monthly variation of the physico-chemical parameters of brackish water from Bori, Rivers State}

\begin{tabular}{|c|c|c|c|c|c|c|c|}
\hline Parameters & January & February & March & April & May & June & W.H.O limits \\
\hline Conductivity $\mu \mathrm{S} / \mathrm{cm}$ & $2125.41 \pm 120.11$ & $2137.55 \pm 64.24$ & $2157.22 \pm 96.40$ & $2479.80 \pm 100.10$ & \begin{tabular}{|l|}
$3377.81 \pm 146.29$ \\
\end{tabular} & $3842.56 \pm 164.00$ & $1200 \mu \mathrm{s} / \mathrm{cm}$ \\
\hline Alkalinity & $211.47 \pm 7.044$ & $215.53 \pm 3.522$ & $211.47 \pm 7.044$ & $225.70 \pm 7.044$ & $219.60 \pm 7.044$ & $205.37 \pm 3.522$ & $100 \mathrm{mg} / \mathrm{L}$ \\
\hline Turbidity & $12.04 \pm 0.125$ & $12.18 \pm 0.157$ & $12.13 \pm 0.157$ & $13.18 \pm 0.303$ & $14.34 \pm 0.496$ & $14.88 \pm 0.637$ & $5.0 \mathrm{NTU}$ \\
\hline Sulphate & $541.74 \pm 2.54$ & $553.41 \pm 2.12$ & $560.08 \pm 1.87$ & $534.41 \pm 2.12$ & $542.74 \pm 1.63$ & $521.41 \pm 2.64$ & $500 \mathrm{mg} / \mathrm{L}$ \\
\hline Total hardness & $404.00 \pm 1.00$ & $403.00 \pm 1.00$ & $403.00 \pm 1.00$ & $396.00 \pm 2.00$ & $390.00 \pm 2.00$ & $406.00 \pm 2.00$ & $100 \mathrm{mg} / \mathrm{L}$ \\
\hline \multirow[t]{2}{*}{ Nitrate } & $2.62 \pm 0.050$ & $2.63 \pm 0.050$ & $2.85 \pm 0.161$ & $2.70 \pm 0.105$ & $3.06 \pm 0.128$ & $2.82 \pm 0.030$ & $5 \mathrm{mg} / \mathrm{L}$ \\
\hline & July & August & September & October & \begin{tabular}{|l|} 
November \\
\end{tabular} & December & \\
\hline Conductivity $\mu \mathrm{S} / \mathrm{cm}$ & $3600.44 \pm 220.96$ & $3429.42 \pm 169.56$ & $3444.76 \pm 119.60$ & $3198.11 \pm 67.82$ & $2720.90 \pm 120.78$ & $2153.57 \pm 63.80$ & $1200 \mu \mathrm{s} / \mathrm{cm}$ \\
\hline Dissolved solids & $2610.21 \pm 160.25$ & $2486.22 \pm 122.78$ & $2497.45 \pm 86.71$ & $2318.63 \pm 49.17$ & $1972.54 \pm 87.57$ & $1561.34 \pm 46.26$ & $500 \mathrm{mg} / \mathrm{L}$ \\
\hline Alkalinity & $205.37 \pm 3.522$ & $203.33 \pm 3.522$ & $223.67 \pm 7.044$ & $217.57 \pm 7.044$ & $217.57 \pm 3.522$ & $211.47 \pm 3.522$ & $100 \mathrm{mg} / \mathrm{L}$ \\
\hline Dissolved oxygen & $4.60 \pm 0.361$ & $4.60 \pm 0.200$ & $4.40 \pm 0.211$ & $4.30 \pm 0.200$ & $3.90 \pm 0.529$ & $3.43 \pm 0.586$ & Not specified \\
\hline Magnesium hardness & $119.00 \pm 1.15$ & $129.00 \pm 1.15$ & $119.00 \pm 1.15$ & $118.00 \pm 0.58$ & $120.00 \pm 0.58$ & $119.00 \pm 0.58$ & Not specified \\
\hline Nitrate & $2.49 \pm 0.039$ & $2.50 \pm 0.076$ & $2.49 \pm 0.090$ & $2.50 \pm 0.076$ & $2.49 \pm 0.071$ & $2.58 \pm 0.087$ & $5 \mathrm{mg} / \mathrm{L}$ \\
\hline
\end{tabular}




\section{Table 2: Monthly variation of the trace element parameters of brackish water from Bori, Rivers State}

\begin{tabular}{|c|c|c|c|c|c|c|c|}
\hline Parameters & January & February & March & April & May & June & W.H.O limits \\
\hline Calcium & $111.42 \pm 0.23$ & $111.02 \pm 0.23$ & $111.02 \pm 0.23$ & $103.41 \pm 0.46$ & $102.60 \pm 0.46$ & $115.43 \pm 0.46$ & $75 \mathrm{mg} / \mathrm{L}$ \\
\hline Magnesium & $30.62 \pm 0.14$ & $30.62 \pm 0.14$ & $30.62 \pm 0.14$ & $33.53 \pm 0.28$ & $32.56 \pm 0.28$ & $28.67 \pm 0.28$ & $20 \mathrm{mg} / \mathrm{L}$ \\
\hline Copper & $0.0200 \pm 0.0017$ & $0.0300 \pm 0.0015$ & $0.0200 \pm 0.0016$ & $0.0400 \pm 0.0020$ & $0.0400 \pm 0.0021$ & $0.0400 \pm 0.0021$ & $2.0 \mathrm{mg} / \mathrm{L}$ \\
\hline Chromium & $0.0036 \pm 0.0003$ & $0.0038 \pm 0.0003$ & $0.0035 \pm 0.0005$ & $0.0036 \pm 0.0005$ & $0.0039 \pm 0.0006$ & $0.0039 \pm 0.0006$ & $0.05 \mathrm{mg} / \mathrm{L}$ \\
\hline Cadmium & $0.0020 \pm 0.0004$ & $0.0025 \pm 0.0002$ & $0.0030 \pm 0.0002$ & $0.0035 \pm 0.0002$ & $0.0045 \pm 0.0002$ & $0.0050 \pm 0.0001$ & $0.003 \mathrm{mg} / \mathrm{L}$ \\
\hline Lead & $0.0040 \pm 0.0007$ & $0.0038 \pm 0.0009$ & $0.0039 \pm 0.0008$ & $0.0033 \pm 0.0010$ & $0.0033 \pm 0.0011$ & $0.0031 \pm 0.0012$ & $0.01 \mathrm{mg} / \mathrm{L}$ \\
\hline Iron & $2.80 \pm 0.113$ & $2.82 \pm 0.121$ & $2.75 \pm 0.090$ & $2.66 \pm 0.438$ & $2.64 \pm 0.654$ & $3.23 \pm 0.592$ & $3 \mathrm{mg} / \mathrm{L}$ \\
\hline \multirow[t]{2}{*}{ Manganese } & $0.0055 \pm 0.0005$ & $0.0055 \pm 0.0005$ & $0.0060 \pm 0.0006$ & $0.0063 \pm 0.0006$ & $0.0061 \pm 0.0007$ & $0.0063 \pm 0.0006$ & $0.4 \mathrm{mg} / \mathrm{L}$ \\
\hline & July & August & September & October & November & December & \\
\hline Calcium & $115.03 \pm 0.46$ & $112.22 \pm 0.46$ & $115.83 \pm 0.46$ & $116.23 \pm 0.23$ & $115.03 \pm 0.23$ & $115.43 \pm 0.23$ & $75 \mathrm{mg} / \mathrm{L}$ \\
\hline Magnesium & $28.92 \pm 0.28$ & $31.35 \pm 0.28$ & $28.92 \pm 0.28$ & $28.67 \pm 0.14$ & $29.16 \pm 0.14$ & $28.92 \pm 0.14$ & $20 \mathrm{mg} / \mathrm{L}$ \\
\hline Copper & $0.0500 \pm 0.0022$ & $0.0500 \pm 0.0024$ & $0.0400 \pm 0.0018$ & $0.0400 \pm 0.0020$ & $0.0300 \pm 0.0021$ & $0.0300 \pm 0.0020$ & $2.0 \mathrm{mg} / \mathrm{L}$ \\
\hline Chromium & $0.0040 \pm 0.0005$ & $0.0035 \pm 0.0005$ & $0.0038 \pm 0.0005$ & $0.0036 \pm 0.0004$ & $0.0035 \pm 0.0004$ & $0.0034 \pm 0.0003$ & $0.05 \mathrm{mg} / \mathrm{L}$ \\
\hline Cadmium & $0.0060 \pm 0.0001$ & $0.0050 \pm 0.0002$ & $0.0050 \pm 0.0001$ & $0.0030 \pm 0.0003$ & $0.0028 \pm 0.0004$ & $0.0026 \pm 0.0001$ & $0.003 \mathrm{mg} / \mathrm{L}$ \\
\hline Lead & $0.0033 \pm 0.0008$ & $0.0028 \pm 0.0011$ & $0.0030 \pm 0.0009$ & $0.0033 \pm 0.0009$ & $0.0036 \pm 0.0008$ & $0.0038 \pm 0.0007$ & $0.01 \mathrm{mg} / \mathrm{L}$ \\
\hline Iron & $3.15 \pm 0.666$ & $3.75 \pm 0.433$ & $3.85 \pm 0.306$ & $3.94 \pm 0.510$ & $3.64 \pm 0.395$ & $2.95 \pm 0.324$ & $3 \mathrm{mg} / \mathrm{L}$ \\
\hline Manganese & $0.0065 \pm 0.0005$ & $0.0063 \pm 0.0005$ & $0.0064 \pm 0.0007$ & $0.0063 \pm 0.0007$ & $0.0060 \pm 0.0009$ & $0.0055 \pm 0.0007$ & $0.4 \mathrm{mg} / \mathrm{L}$ \\
\hline
\end{tabular}




\section{Table 3. Monthly variation of the physico-chemical parameters of brackish water from Okoli'ile, Rivers State}

\begin{tabular}{|c|c|c|c|c|c|c|c|}
\hline Parameters & January & February & March & April & May & June & W.H.O limits \\
\hline Conductivity $\mu \mathrm{S} / \mathrm{cm}$ & $2032.63 \pm 34.47$ & $2093.28 \pm 78.37$ & $2062.82 \pm 72.90$ & $2438.79 \pm 50.09$ & $3124.75 \pm 228.82$ & $3588.94 \pm 163.50$ & $1200 \mu \mathrm{s} / \mathrm{cm}$ \\
\hline Dissolved solids & $1473.65 \pm 24.99$ & $1517.58 \pm 56.81$ & $1495.54 \pm 52.86$ & $1768.13 \pm 36.32$ & $2265.44 \pm 165.89$ & $2601.98 \pm 118.54$ & $500 \mathrm{mg} / \mathrm{L}$ \\
\hline Alkalinity & $164.70 \pm 6.100$ & $172.83 \pm 3.522$ & $183.00 \pm 6.100$ & $178.93 \pm 3.522$ & $187.07 \pm 3.522$ & $162.67 \pm 3.522$ & $100 \mathrm{mg} / \mathrm{L}$ \\
\hline Dissolved oxygen & $3.42 \pm 0.104$ & $3.28 \pm 0.076$ & $3.22 \pm 0.0 .257$ & $3.78 \pm 0.749$ & $4.25 \pm 0.203$ & $5.02 \pm 0.257$ & Not specified \\
\hline Turbidity & $11.24 \pm 0.210$ & $11.23 \pm 0.0 .259$ & $11.99 \pm 0.069$ & $12.65 \pm 0.203$ & $13.71 \pm 0.246$ & $13.04 \pm 360$ & $5.0 \mathrm{NTU}$ \\
\hline Sulphate & $373.58 \pm 3.71$ & $377.89 \pm 2.15$ & $396.30 \pm 1,79$ & $400.18 \pm 1.87$ & $394.56 \pm 1.68$ & $385.60 \pm 2.5$ & $500 \mathrm{mg} / \mathrm{L}$ \\
\hline Total hardness & $400.00 \pm 1.00$ & $399.00 \pm 1.00$ & $398.00 \pm 1.00$ & $392.00 \pm 2.00$ & $391.00 \pm 2.00$ & $403.00 \pm 2.00$ & $100 \mathrm{mg} / \mathrm{L}$ \\
\hline Calcium hardness & $272.00 \pm 0.58$ & $270.00 \pm 0.58$ & $271.00 \pm 0.58$ & $265.00 \pm 1.15$ & $264.00 \pm 1.15$ & $274.00 \pm 1.15$ & Not specified \\
\hline Magnesium hardness & $128.00 \pm 0.58$ & $129.00 \pm 0.58$ & $127.00 \pm 0.58$ & $127.00 \pm 1.15$ & $127.00 \pm 1.15$ & $129.00 \pm 1.15$ & Not specified \\
\hline \multirow[t]{2}{*}{ Nitrate } & $3.61 \pm 0.060$ & $3.72 \pm 0.062$ & $3.61 \pm 0.068$ & $3.69 \pm 0.072$ & $3.81 \pm 0.091$ & $3.70 \pm 0.136$ & $5 \mathrm{mg} / \mathrm{L}$ \\
\hline & July & August & September & October & November & December & \\
\hline Conductivity $\mu \mathrm{S} / \mathrm{cm}$ & $3816.23 \pm 117.67$ & $3296.01 \pm 45.70$ & $3426.24 \pm 125.78$ & $3166.28 \pm 49.34$ & $2860.13 \pm 46.66$ & $2179.99 \pm 60.41$ & $1200 \mu \mathrm{s} / \mathrm{cm}$ \\
\hline Dissolved solids & $2766.77 \pm 85.31$ & $2389.64 \pm 33.08$ & $2484.02 \pm 91.20$ & $2295.55 \pm 35.77$ & $2073.59 \pm 33.83$ & $1580.44 \pm 43.88$ & $500 \mathrm{mg} / \mathrm{L}$ \\
\hline Alkalinity & $154.53 \pm 9.318$ & $162.67 \pm 3.522$ & $156.57 \pm 3.522$ & $166.73 \pm 7.044$ & $162.67 \pm 3.522$ & $156.67 \pm 3.522$ & $100 \mathrm{mg} / \mathrm{L}$ \\
\hline Dissolved oxygen & $5.02 \pm 0.257$ & $4.58 \pm 0.369$ & $4.78 \pm 0.076$ & $4.82 \pm 0.104$ & $4.65 \pm 0.087$ & $4.02 \pm 0.126$ & Not specified \\
\hline Turbidity & $12.75 \pm 0.169$ & $12.55 \pm 0.176$ & $12.23 \pm 0.210$ & $11.77 \pm 0.263$ & $11.58 \pm 0.165$ & $11.42 \pm 0.188$ & $5.0 \mathrm{NTU}$ \\
\hline Sulphate & $378.23 \pm 1.84$ & $383.25 \pm 1.95$ & $376.44 \pm 1.72$ & $372.85 \pm 2.64$ & $366.23 \pm 3.01$ & $363.56 \pm 2.55$ & $500 \mathrm{mg} / \mathrm{L}$ \\
\hline Total hardness & $404.00 \pm 2.00$ & $410.00 \pm 1.00$ & $409.00 \pm 2.00$ & $409.00 \pm 2.00$ & $400.00 \pm 1.00$ & $399.00 \pm 1.00$ & $100 \mathrm{mg} / \mathrm{L}$ \\
\hline Calcium hardness & $275.00 \pm 1.15$ & $280.00 \pm 0.58$ & $278.00 \pm 1.15$ & $280.00 \pm 1.15$ & $273.00 \pm 0.58$ & $271.00 \pm 0.58$ & Not specified \\
\hline Magnesium hardness & $129.00 \pm 1.15$ & $130.00 \pm 0.58$ & $131.00 \pm 1.15$ & $129.00 \pm 1.15$ & $127.00 \pm 0.58$ & $128.00 \pm 0.58$ & Not specified \\
\hline Nitrate & $3.60 \pm 0.126$ & $3.65 \pm 0.151$ & $3.59 \pm 0.250$ & $3.75 \pm 0.2000$ & $3.66 \pm 0.070$ & $3.50 \pm 0.145$ & $5 \mathrm{mg} / \mathrm{L}$ \\
\hline
\end{tabular}




\section{Table 4. Monthly variation of the trace element parameters of brackish water from Okoli'ile, Rivers State}

\begin{tabular}{|c|c|c|c|c|c|c|c|}
\hline Parameters & January & February & March & April & May & June & $\begin{array}{l}\text { W.H.O } \\
\text { limits }\end{array}$ \\
\hline Calcium & $109.02 \pm 0.23$ & $108.22 \pm 0.23$ & $108.62 \pm 0.23$ & $106.21 \pm 0.46$ & $105.81 \pm 0.46$ & $109.82 \pm 0.46$ & $75 \mathrm{mg} / \mathrm{L}$ \\
\hline Magnesium & $31.10 \pm 0.14$ & $31.35 \pm 0.14$ & $30.86 \pm 0.14$ & $30.86 \pm 0.28$ & $30.86 \pm 0.28$ & $31.35 \pm 0.28$ & $20 \mathrm{mg} / \mathrm{L}$ \\
\hline Zinc & $0.0041 \pm 0.0008$ & $0.0440 \pm 0.0008$ & $0.0480 \pm 0.0008$ & $0.0510 \pm 0.0007$ & $0.0550 \pm 0.0008$ & $0.0066 \pm 0.0009$ & $3.0 \mathrm{mg} / \mathrm{L}$ \\
\hline Copper & $0.0200 \pm 0.0038$ & $0.0300 \pm 0.0035$ & $0.0300 \pm 0.0036$ & $0.0400 \pm 0.0036$ & $0.0400 \pm 0.0037$ & $0.0500 \pm 0.0030$ & $2.0 \mathrm{mg} / \mathrm{L}$ \\
\hline Chromium & $0.0038 \pm 0.0001$ & $0.0039 \pm 0.0001$ & $0.0036 \pm 0.0003$ & $0.0035 \pm 0.0004$ & $0.0039 \pm 0.0003$ & $0.0040 \pm 0.0003$ & $0.05 \mathrm{mg} / \mathrm{L}$ \\
\hline Cadmium & $0.0030 \pm 0.0002$ & $0.0030 \pm 0.0002$ & $0.0040 \pm 0.0001$ & $0.0048 \pm 0.0002$ & $0.0050 \pm 0.0002$ & $0.0060 \pm 0.0003$ & $0.003 \mathrm{mg} / \mathrm{L}$ \\
\hline Lead & $0.0040 \pm 0.0005$ & $0.0038 \pm 0.0005$ & $0.0040 \pm 0.0005$ & $0.0035 \pm 0.0006$ & $0.0035 \pm 0.0005$ & $0.0031 \pm 0.0007$ & $0.01 \mathrm{mg} / \mathrm{L}$ \\
\hline Iron & $3.31 \pm 0.269$ & $3.54 \pm 0.194$ & $3.64 \pm 0.196$ & $3.76 \pm 0.215$ & $4.79 \pm 0.883$ & $5.06 \pm 1.178$ & $3 \mathrm{mg} / \mathrm{L}$ \\
\hline \multirow[t]{2}{*}{ Manganese } & $0.0056 \pm 0.0006$ & $0.0055 \pm 0.0006$ & $0.0060 \pm 0.0006$ & $0.0065 \pm 0.0008$ & $0.0066 \pm 0.0008$ & $0.0069 \pm 0.0009$ & $0.4 \mathrm{mg} / \mathrm{L}$ \\
\hline & July & August & September & October & November & December & \\
\hline Calcium & $110.22 \pm 0.46$ & $112.22 \pm 0.46$ & $111.42 \pm 0.46$ & $112.22 \pm 0.46$ & $109.42 \pm 0.23$ & $108.62 \pm 0.23$ & $75 \mathrm{mg} / \mathrm{L}$ \\
\hline Magnesium & $31.35 \pm 0.28$ & $30.86 \pm 0.28$ & $31.83 \pm 0.28$ & $31.35 \pm 0.28$ & $30.86 \pm 0.14$ & $31.10 \pm 0.14$ & $20 \mathrm{mg} / \mathrm{L}$ \\
\hline Zinc & $0.0620 \pm 0.0011$ & $0.0590 \pm 0.0014$ & $0.0600 \pm 0.0018$ & $0.0560 \pm 0.0016$ & $0.0550 \pm 0.0010$ & $0.0480 \pm 0.0008$ & $3.0 \mathrm{mg} / \mathrm{L}$ \\
\hline Copper & $0.0600 \pm 0.0032$ & $0.0500 \pm 0.0033$ & $0.0400 \pm 0.0030$ & $0.0400 \pm 0.0031$ & $0.0300 \pm 0.0033$ & $0.0300 \pm 0.0031$ & $2.0 \mathrm{mg} / \mathrm{L}$ \\
\hline Chromium & $0.0043 \pm 0.0002$ & $0.0044 \pm 0.0001$ & $0.0039 \pm 0.0003$ & $0.0038 \pm 0.0004$ & $0.0035 \pm 0.0003$ & $0.0037 \pm 0.0004$ & $0.05 \mathrm{mg} / \mathrm{L}$ \\
\hline Cadmium & $0.0060 \pm 0.0002$ & $0.0060 \pm 0.0002$ & $0.0050 \pm 0.0003$ & $0.0040 \pm 0.0002$ & $0.0038 \pm 0.0002$ & $0.0029 \pm 0.0003$ & $0.003 \mathrm{mg} / \mathrm{L}$ \\
\hline Lead & $0.0033 \pm 0.0005$ & $0.0027 \pm 0.0006$ & $0.0030 \pm 0.0005$ & $0.0034 \pm 0.0005$ & $0.0036 \pm 0.0005$ & $0.0036 \pm 0.0004$ & $0.01 \mathrm{mg} / \mathrm{L}$ \\
\hline Iron & $4.99 \pm 1.218$ & $5.01 \pm 1.173$ & $4.80 \pm 0.990$ & $4.66 \pm 0.763$ & $3.78 \pm 0.218$ & $3.49 \pm 0.171$ & $3 \mathrm{mg} / \mathrm{L}$ \\
\hline Manganese & $0.0065 \pm 0.0008$ & $0.0063 \pm 0.0009$ & $0.0066 \pm 0.0007$ & $0.0063 \pm 0.0006$ & $0.0060 \pm 0.0005$ & $0.0057 \pm 0.0006$ & $0.4 \mathrm{mg} / \mathrm{L}$ \\
\hline
\end{tabular}




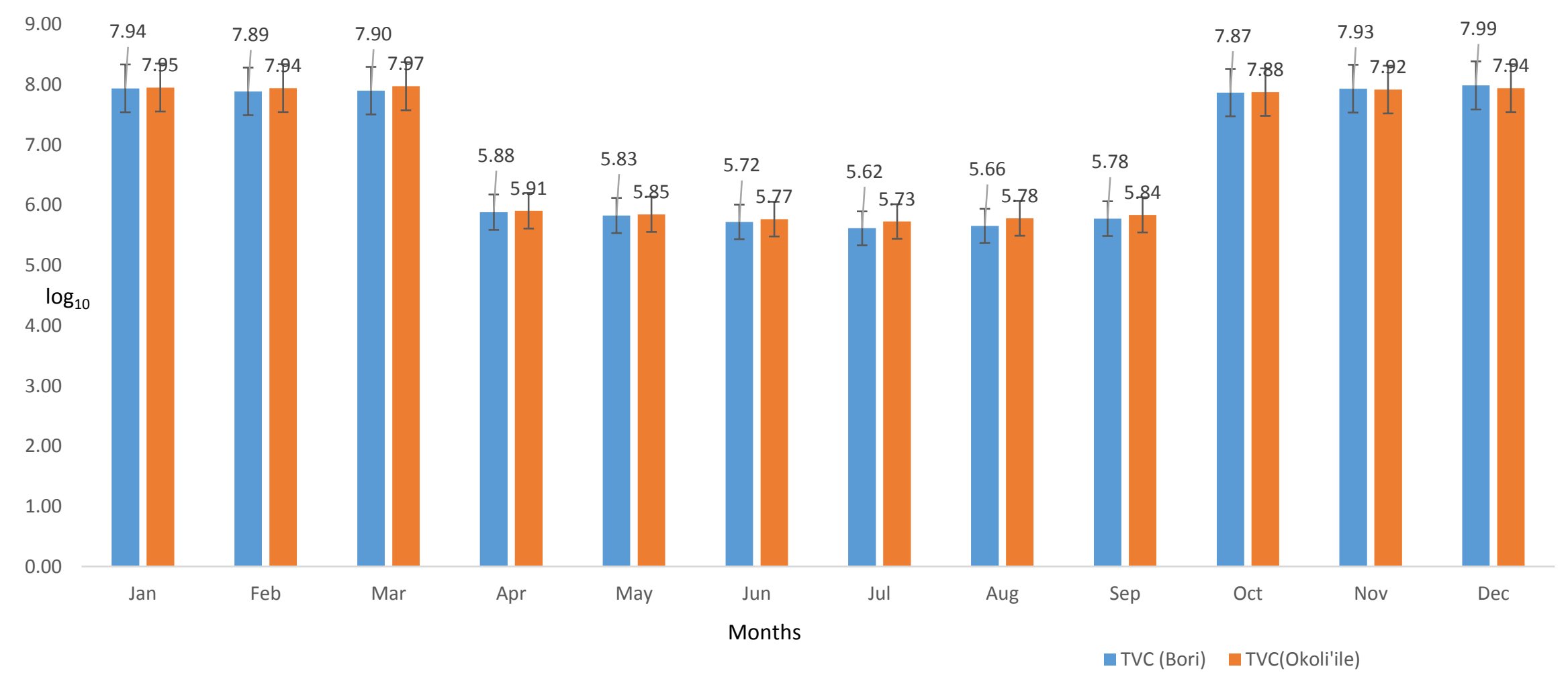

Figure 5. Monthly mean values $\left(\log _{10}\right)$ of total heterotrophic count $(\mathrm{cfu} / \mathrm{ml})$ of brackish water from Okoli'ile, Rivers State 


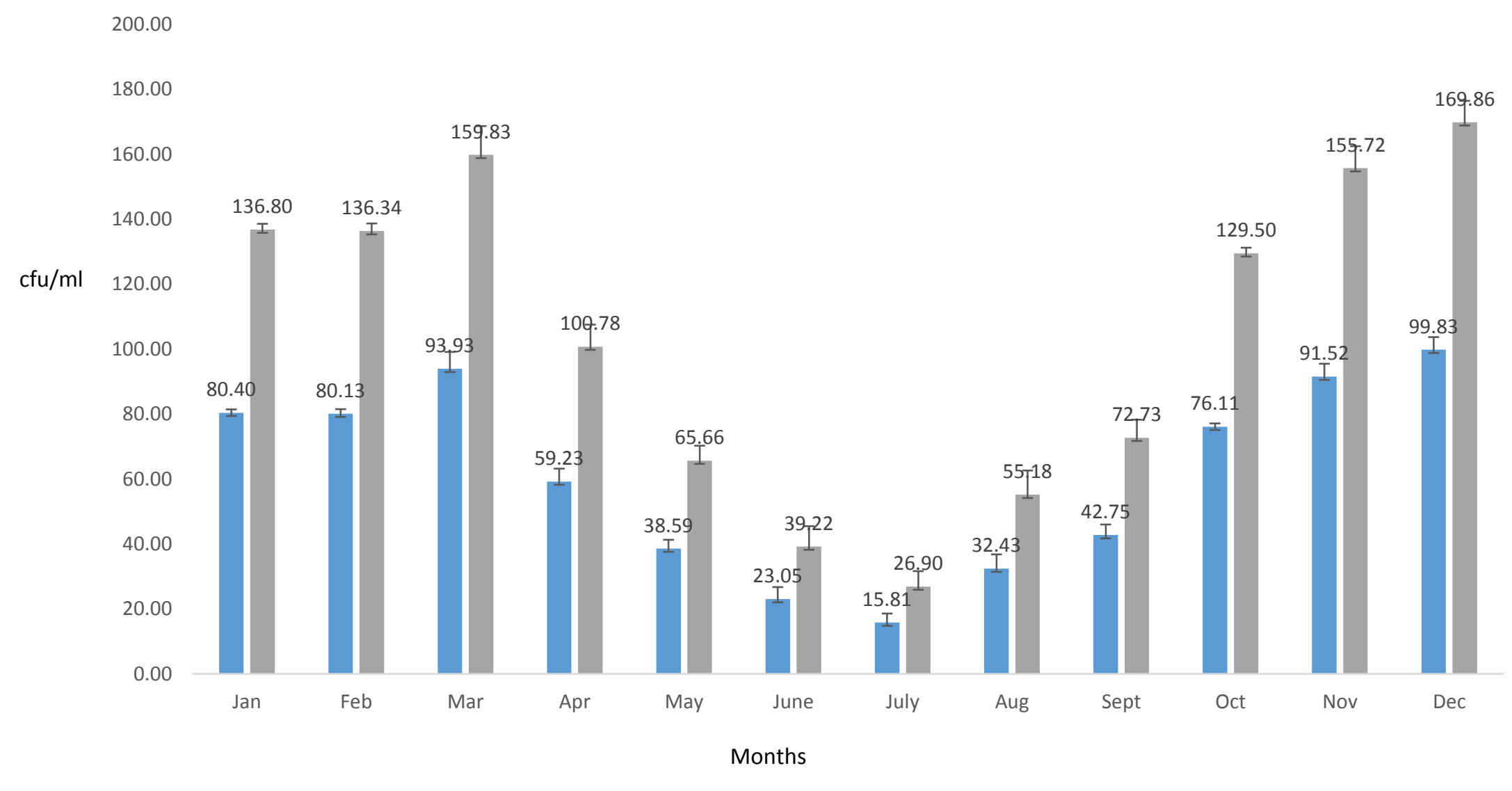

Figure 6. Monthly mean values of total coliform count (cfu/ml) of brackish water from Okoli’ile, Rivers State 


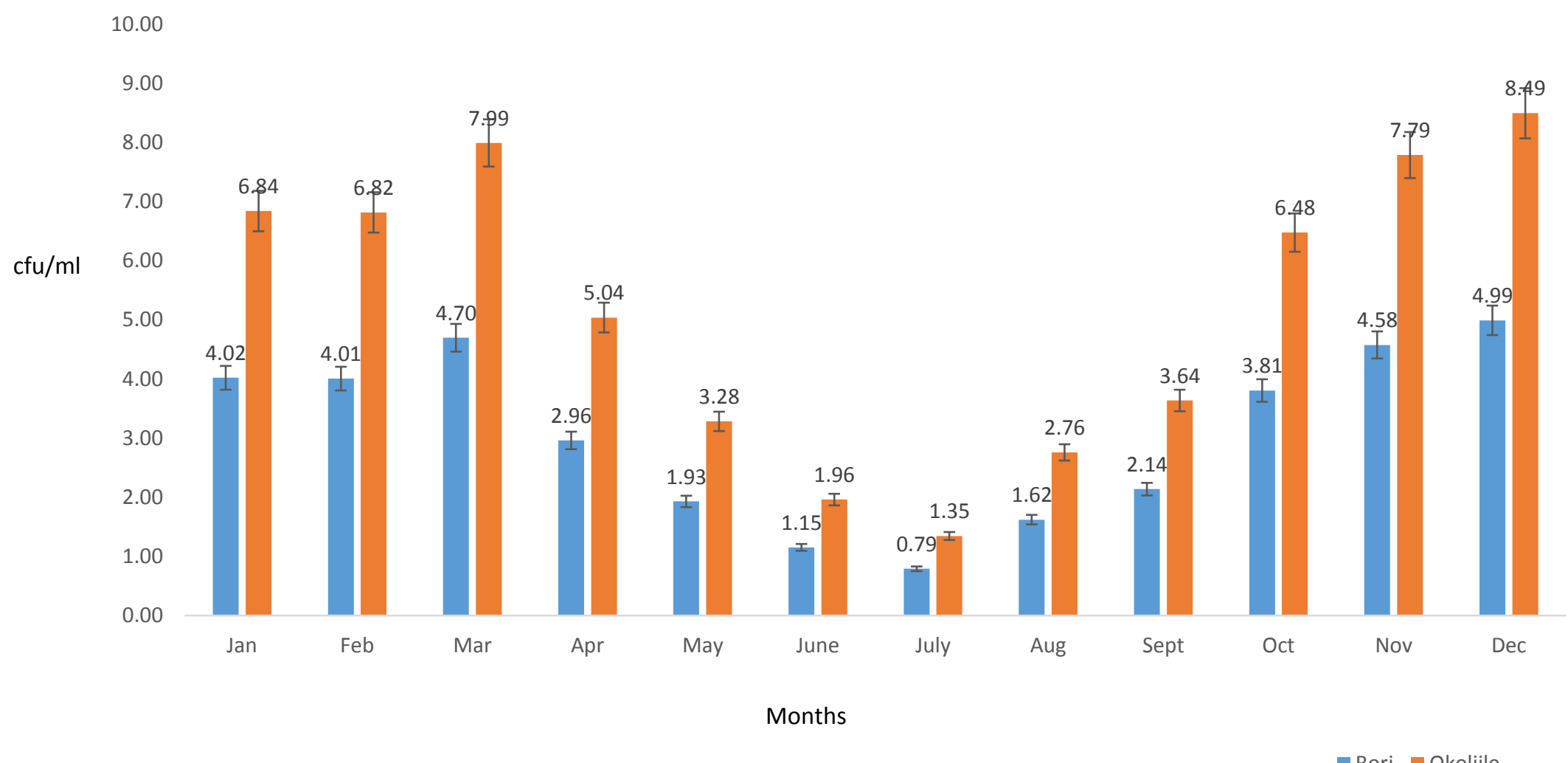

Figure 7. Monthly mean values of faecal coliform count (cfu/ml) of brackish water from Okoli'ile, Rivers State 
Table 5: Monthly mean values of Enterococcus faecalis (CFU/ml) count of brackish water from Bori and Okoli'ile, Rivers State.

\begin{tabular}{|l|l|l|}
\hline & Bori & Okoli'ile \\
\hline Jan & $1.33 \pm 0.58$ & $1.90 \pm 0.70$ \\
\hline Feb & $3.00 \pm 1.00$ & $3.60 \pm 1.10$ \\
\hline Mar & $3.00 \pm 1.00$ & $3.60 \pm 1.10$ \\
\hline Apr & $1.67 \pm 0.58$ & $2.20 \pm 0.70$ \\
\hline May & $1.33 \pm 0.58$ & $1.90 \pm 0.70$ \\
\hline Jun & $1.33 \pm 0.58$ & $1.60 \pm 1.10$ \\
\hline Jul & $1.00 \pm 0.58$ & $1.20 \pm 0.70$ \\
\hline Aug & $1.00 \pm 0.58$ & $1.20 \pm 0.70$ \\
\hline Sep & $1.00 \pm 0.58$ & $1.60 \pm 1.10$ \\
\hline Oct & $2.33 \pm 0.58$ & $2.90 \pm 0.70$ \\
\hline Nov & $3.00 \pm 1.00$ & $3.60 \pm 1.10$ \\
\hline Dec & $2.67 \pm 0.58$ & $3.20 \pm 0.70$ \\
\hline
\end{tabular}

Table 6. Pearson's correlation coefficients of indicator microorganisms and physicochemical parameters of surface water from Bori in Rivers State, Nigeria

\begin{tabular}{|c|c|c|c|c|c|c|c|}
\hline \multirow{4}{*}{ TVC } & & TVC & $\mathrm{TCC}$ & $\mathrm{TFC}$ & $\mathrm{pH}$ & Temp & Salinity \\
\hline & Pearson Correlation & 1.000 & $.932^{* *}$ & $.932^{* *}$ & .468 & .470 & -.198 \\
\hline & Sig. (2-tailed) & & .000 & .000 & .125 & .123 & .538 \\
\hline & $\mathrm{N}$ & 12 & 12 & 12 & 12 & 12 & 12 \\
\hline \multirow[t]{3}{*}{ TCC } & Pearson Correlation & $.932^{* *}$ & 1.000 & $1.000^{* *}$ & .483 & .544 & -.108 \\
\hline & Sig. (2-tailed) & .000 & & .000 & .112 & .067 & .738 \\
\hline & $\mathrm{N}$ & 12 & 12 & 12 & 12 & 12 & 12 \\
\hline \multirow[t]{3}{*}{ TFC } & Pearson Correlation & $.932^{* *}$ & $1.000^{* *}$ & 1.000 & .482 & .544 & -.108 \\
\hline & Sig. (2-tailed) & .000 & .000 & & .112 & .068 & .738 \\
\hline & $\mathrm{N}$ & 12 & 12 & 12 & 12 & 12 & 12 \\
\hline \multirow[t]{3}{*}{$\overline{\mathrm{pH}}$} & Pearson Correlation & .468 & .483 & .482 & 1.000 & $.949^{* *}$ & .575 \\
\hline & Sig. (2-tailed) & .125 & .112 & .112 & & .000 & .051 \\
\hline & $\mathrm{N}$ & 12 & 12 & 12 & 12 & 12 & 12 \\
\hline \multirow[t]{3}{*}{ Temp } & Pearson Correlation & .470 & .544 & .544 & $.949^{* *}$ & 1.000 & $.587^{*}$ \\
\hline & Sig. (2-tailed) & .123 & .067 & .068 & .000 & & .045 \\
\hline & $\mathrm{N}$ & 12 & 12 & 12 & 12 & 12 & 12 \\
\hline \multirow[t]{3}{*}{ Salinity } & Pearson Correlation & -.198 & -.108 & -.108 & .575 & $.587^{*}$ & 1.000 \\
\hline & Sig. (2-tailed) & .538 & .738 & .738 & .051 & .045 & \\
\hline & $\mathrm{N}$ & 12 & 12 & 12 & 12 & 12 & 12 \\
\hline
\end{tabular}

TVC: Total heterotrophic count; TCC: Total coliform count; TFC: Total faecal count; 
Table 7. Pearson's correlation coefficients of indicator microorganisms and physicochemical parameters of surface water from Okoli'ile in Rivers State, Nigeria.

\begin{tabular}{|c|c|c|c|c|c|c|c|}
\hline \multirow{4}{*}{ TVC } & & TVC & TCC & TFC & $\mathrm{pH}$ & Temp & Salinity \\
\hline & Pearson Correlation & 1.000 & $.927^{* *}$ & $.927^{* *}$ & $.704^{*}$ & .493 & $.608^{*}$ \\
\hline & Sig. (2-tailed) & & .000 & .000 & .011 & .103 & .036 \\
\hline & $\mathrm{N}$ & 12 & 12 & 12 & 12 & 12 & 12 \\
\hline \multirow[t]{3}{*}{ TCC } & Pearson Correlation & $.927^{* *}$ & 1.000 & $1.000^{* *}$ & $.690^{*}$ & .567 & $.670^{*}$ \\
\hline & Sig. (2-tailed) & .000 & & .000 & .013 & .054 & .017 \\
\hline & $\mathrm{N}$ & 12 & 12 & 12 & 12 & 12 & 12 \\
\hline \multirow[t]{3}{*}{ TFC } & Pearson Correlation & $.927^{* *}$ & $1.000^{* *}$ & 1.000 & $.690^{*}$ & .567 & $.669^{*}$ \\
\hline & Sig. (2-tailed) & .000 & .000 & & .013 & .055 & .017 \\
\hline & $\mathrm{N}$ & 12 & 12 & 12 & 12 & 12 & 12 \\
\hline \multirow[t]{3}{*}{$\mathrm{pH}$} & Pearson Correlation & $.704^{*}$ & $.690^{*}$ & $.690^{*}$ & 1.000 & $.825^{* *}$ & $.860^{* *}$ \\
\hline & Sig. (2-tailed) & .011 & .013 & .013 & & .001 & .000 \\
\hline & $\mathrm{N}$ & 12 & 12 & 12 & 12 & 12 & 12 \\
\hline \multirow[t]{3}{*}{ Temp } & Pearson Correlation & .493 & .567 & .567 & $.825^{* *}$ & 1.000 & $.951^{* *}$ \\
\hline & Sig. (2-tailed) & .103 & .054 & .055 & .001 & & .000 \\
\hline & $\mathrm{N}$ & 12 & 12 & 12 & 12 & 12 & 12 \\
\hline \multirow[t]{3}{*}{ Salinity } & Pearson Correlation & $.608^{*}$ & $.670^{*}$ & $.669^{*}$ & $.860^{* *}$ & $.951^{* *}$ & 1.000 \\
\hline & Sig. (2-tailed) & .036 & .017 & .017 & .000 & .000 & \\
\hline & $\mathrm{N}$ & 12 & 12 & 12 & 12 & 12 & 12 \\
\hline
\end{tabular}

TVC: Total heterotrophic count; TCC: Total coliform count; TFC: Total faecal count;

\section{DISCUSSION}

The result of the physicochemical analysis of surface waters in Bori and Okoli'ile showed similarities in the data obtained over the duration of 12 months. The $\mathrm{pH}$ values peaked at Bori (7.38) and Okoli'ile (7.29). It was observed that the values obtained from these two localities had figures for $\mathrm{pH}$ values within the acceptable level for environmental tolerance and drinking (6.5-8.5). The values of $\mathrm{pH}$ observed in this study showed a similar trend with reported studies in Calabar river (Akpan, 2000; Wetzel, 2001). It was observed that average $\mathrm{pH}$ values were considerably reduced in the month of April to October, a period where the rain is abundant and a period known as the rainy season or wet months in tropical sub-saharan Africa. The trend observed in the decline in the $\mathrm{pH}$ values in wet months is in agreement with the reports of Ajao and Fagade (2002) and Udoh et al. (2013). Similar occurrence of monthly distribution was observed in the values of temperature $\left({ }^{0} \mathrm{C}\right)$ where lower average values were also observed in wet months. Salinity is the measurement of salt concentration in $\mathrm{mg} / \mathrm{L}$. The result of salinity in this study showed higher values in Bori with statistically significant difference at $\mathrm{P}<0.05$. In addition, the values of salt concentration were lower in the wet months. The likely reasons for these reductions of mean values for $\mathrm{pH}$, temperature and salinity in the wet months could be attributed to higher discharge of fresh water during these months when rain is frequent (Abowei, 2010; Udoh et al., 2013). 
The result of physicochemical parameters of brackish water (table 1) and trace elements (table 2) from Bori showed similar trends of results with data from physicochemical parameters of brackish water (table 3) and trace elements (table 4) from Okoli'ile. With the exception of nitrates, all other physicochemical indices of water quality assessment exceeded the environmentally acceptable limits. Saliu and Ekpo (2006) asserted that the indiscriminate disposal of human and industrial wastes into creeks and lagoons is the major reason the quality of water and aesthetics is diminished. Their assertion was further confirmed in studies of Onyema, 2013. The high values of iron concentration exceeding limits - in some months in Bori and every month in Okoli'ile - could be due to the activities of oil-exploration companies in the area with attendant spills.

The microbiological examination of surface waters from both localities revealed lowered total heterotrophic counts in the wet months (April-September). There were significant differences $(\mathrm{P}<0.05)$ in the values of total viable counts in the dry months and in the wet months. This could be attributed to the fact that during these wet months, there was an increased influx of fresh water into the water body (Onyegeme-Okerenta et al., 2016). Similar occurrence was observed in total coliform (figure 6) and faecal coliform (figure 7) with statistically significant $(\mathrm{P}<0.05)$ higher mean values during the dry seasons when there were lesser rainfalls. In table 5, the monthly mean values of E. faecalis were higher in Okoli'ile with statistical difference $(\mathrm{P}<0.05)$ in the values recorded. The average monthly values of these indicator microorganisms, which had higher distribution in the dry months from the two localities with statistically significant differences, is in concordance with past reports (Aladese \& Ariyo, 2017; Bello et al., 2013).

\section{CONCLUSION}

It was observed in this study that most of the households in these two settlements tend to channel most of their human excreta through polyvinyl chloride (PVC) pipes directly into the nearest creeks and rivers; a practice that is very prominent in many creek settlements of the Niger-Delta region. Akoachere et al. (2008) linked the inadequacy and absence of effective human waste disposal systems as the major reasons for high contamination of potentially pathogenic microorganisms into water bodies. Indiscriminate dumping of human and industrial wastes into these water bodies have a direct effect on the local population because these inhabitants have regular contacts with these surface water, mostly through drinking and recreational activities and these, in our opinion, could be a predisposing factor for epidemics associated with the region.

\section{RECOMMENDATIONS}

The local and regional governments in these areas are advised to make provision for effective waste treatment and disposal systems, so as to avoid uncontrolled disposal of wastes directly into the water body. In addition, industries operating in these areas should be monitored and encouraged to pre-treat their effluents before they are discharged into the aquatic environment. 


\section{REFERENCES}

Abowei, J. F. N. (2010). Salinity, Dissolved Oxygen, pH and Surface Water Temperature conditions in Nkoro River, Niger Delta, Nigeria. Advanced Journal of Food Science and Technology, 2 (1): 36 -40.

Ademoroti, C.M.A. (1997). Standard methods for water and effluents analysis, $2^{\text {nd }}$ edition. Folude Press Publication, Ibadan, Nigeria. Pp. 20-145.

Ajao, E. A. and Fagade, S. O. (2002). The ecology of Neritina glabrata in Lagos Lagoon, Nigeria. Archive of Hyrobiology, 119(3):339-350.

Akoachere, J.T.K., Oben, P. M., Mbivnjo, B. S., Ndip, L. M., Nkwelang, G. and Ndi, R. N. (2008). Bacterial indicators of pollution of the Douala lagoon, Cameroon: Public health implications. Journal of African Health Science, 8(2):85-89.

Akpan, E. R. (2000). Influence of Meteorological and Hydrographic Factors in the Water Quality of Calabar River, Nigeria. Tropical Journal of Environmental Research, 2 \& 3:107-111.

Aladese, M.A. and Ariyo, A.B. (2017). Physico-Chemical Properties and Bacteria Qualities of hand-dug wells in Ebukuma, Rivers State Nigeria. International Journal of Basic Science and Technology, 3(1):59-72

American Public Health Association (APHA) (1992). Standard methods the examination of water and wastewaters, 18th ed., Washington, D.C

American Public Health Association (APHA) (2005). Standard methods the examination of water and wastewaters, 21 st ed., Washington, D.C

Bello, O.O., Osho, A.; Bankole, S.A. and Bello, T.K. (2013). Bacteriological and physicochemical analyses of borehole and well water sources in Ijebu-Ode, South-western Nigeria. International Journal of Pharmaceutical and Biological Science, 8:18-25.

Cheesbrough, M. (2006). Laboratory practice in tropical countries. 2nd edition, Cambridge university press, 146-153

Diersong, N. (2009). Water Quality; Frequently Asked Questions. Florida Brooks National Marine Sanctuary, Key West. pp. 1-2.

Higler, L. W. G. (2012). Fresh surface water: Biology and Biodiversity of River systems, Encyclopedia of Life Support Systems (EOLSS), International Journal of Innovative Research in Science, Engineering and Technology, 1(3):49-57

Jay, M. O., Banigbo, O., Odukoya, O. O. \& Arowolo, T. A. (2007). Water Quality Assessment of Ogun River, South West Nigeria. Journal of Environmental Monitoring and Assessment, 133(3): 473-482.

NBS. (2012). Assessment of physico-chemical properties of water of Gorai river at Kushtia town in 2014: a case study. Journal of Science Technology Environment and Information, 2(2): 51-60.

Okeola, F.O., Kolawole, O.D., and Ameen, O.M. (2010). Comparative Study of Physicochemical Parameters of Water from a River and its Surrounding Wells for Possible Interactive Effect. Advances in Environmental Biology, 4(3): 336- 340.

Onyegeme-Okerenta B.M., Obia,, C., Wegwu, M.O. (2016). Physicochemical properties of water quality of Imeh, Edegelem and Chokocho communities located along Otamirioche River in Etche Ethnic Nationality of Rivers State. Nigerian Journal of Applied Science and Environmental. Management, 20(1) 113-119.

Onyema, I.C. (2013). The Physico-Chemical Characteristics and Phytoplankton of the Onijedi Lagoon, Lagos. Nature and Science, 11(1):127-135. 
Oyinloye, A.O. and Jegede, G.O. (2004). Geophysical Survey, Geochemical and Microbiology Investigation of ground well water in Ado-Ekiti, North, South Western Nigeria. Global Journal of Geological Science, 2(2): 235-242.

Saliu, J.K. and Ekpo, M.P. (2006). Preliminary chemical and biological assessment of Ogbe creek Lagos, Nigeria. West African Journal of Applied Ecology 9:12-18.

Udoh, J.P., Ukpatu, J.E. and Otoh, A.J. (2013). Spatial Variation in Physico-Chemical Parameters of Eastern Obolo Estuary, Niger Delta, Nigeria. Journal of Environment and Earth Science, 3(12): 163-171.

Wetzel, R. G. (2001). Limnology: Lake and River ecosystems, 3rd edn. Academic Press, New York, 1006pp 